\title{
Immunohistochemical localisation of advanced glycation end products in pulmonary fibrosis
}

\author{
Takeshi Matsuse, Eijiro Ohga, Shinji Teramoto, Masashi Fukayama, Ryoji Nagai, \\ Seikoh Horiuchi, Yasuyoshi Ouchi
}

\begin{tabular}{|c|}
\hline $\begin{array}{l}\text { Department of } \\
\text { Geriatrics, Faculty of } \\
\text { Medicine, University } \\
\text { of Tokyo, Tokyo, Japan } \\
\text { T Matsuse } \\
\text { E Ohga } \\
\text { S Teramoto } \\
\text { Y Ouchi }\end{array}$ \\
\hline $\begin{array}{l}\text { Department of } \\
\text { Pathology, Jichi } \\
\text { Medical School, School } \\
\text { of Medicine, Tochigi, } \\
\text { Japan } \\
\text { M Fukayama }\end{array}$ \\
\hline
\end{tabular}

The Second

Department of

Biochemistry,

Kumamoto University

School of Medicine,

Kumamoto, Japan

R Nagai

S Horiuchi

Correspondence to:

Dr Takeshi Matsuse,

Department of Geriatrics,

Faculty of Medicine,

University of Tokyo, 7-3-1

Hongo Bunkyo-ku, Tokyo

113, Japan.

Accepted for publication 27 March 1998

Table 1 Summary of 6D12 immunoreactivity of pulmonary tissue from cases of pulmonary fibrosis and cases with normal pulmonary parenchyma

\begin{tabular}{|c|c|c|c|c|}
\hline Case & $\begin{array}{l}\text { Age } \\
\text { (years) }\end{array}$ & Macrophages* & $\begin{array}{l}\text { Bronchiolar or } \\
\text { metaplastic epithelium }\end{array}$ & $\begin{array}{l}\text { Hyperplastic smooth muscle } \\
\text { cells andlor interstitium }\end{array}$ \\
\hline$\overline{\mathrm{DAD} 1}$ & 82 & ++ & + & $\mathrm{NE}$ \\
\hline DAD 2 & 75 & + & ++ & $\mathrm{NE}$ \\
\hline DAD 3 & 68 & + & - & $\mathrm{NE}$ \\
\hline IPF 1 & 78 & ++ & + & \pm \\
\hline IPF 2 & 69 & ++ & + & + \\
\hline IPF 3 & 74 & ++ & - & - \\
\hline IPF 4 & 82 & ++ & \pm & + \\
\hline IPF 5 & 74 & ++ & + & \pm \\
\hline IPF 6 & 73 & ++ & \pm & \pm \\
\hline IPF 7 & 53 & ++ & ++ & + \\
\hline Ctrl 1 & 62 & ++ & - & - \\
\hline Ctrl 2 & 37 & - & - & - \\
\hline Ctrl 3 & 71 & - & - & - \\
\hline Ctrl 4 & 30 & + & - & - \\
\hline Ctrl 5 & 62 & - & - & - \\
\hline Ctrl 6 & 61 & - & - & - \\
\hline Ctrl 7 & 48 & - & - & - \\
\hline
\end{tabular}

Grading of immunoreactivity (see Methods): -, negative or very weak; \pm , faintly positive; + positive; ++ strongly positive.

Ctrl, control; DAD, diffuse alveolar damage; IPF, idiopathic pulmonary fibrosis; NE, not evaluated.

${ }^{\star}$ Tissue samples of cases with IPF showed increased expression of 6D12 immunoreactivity $v$ control subjects $(\mathrm{p}<0.05$, Mann-Whitney rank test). proteins in several human and animal tissues and suggest that AGE modification may be involved in the pathogenesis of several diseases including human diabetic complications and atherosclerosis..$^{2-4}$

Pulmonary fibrosis is characterised by a complex process involving chronic inflammation, fibroblast proliferation, and abnormal deposition of interstitial collagen. The key cellular participants in the pathogenesis of pulmonary fibrosis have not been completely determined. However, lung macrophages are assumed to be one of the key cellular participants in these fibroproliferative processes.

AGE modified proteins accumulate in aging extracellular matrix and it is thought that they are taken up by receptors or binding sites on macrophages. The receptor for advanced glycation end products (RAGE) has been supposed to mediate monocyte activation; it has been shown to stimulate monocytes/ macrophages to synthesise and release inflammatory cytokines, in particular tumour necrosis factor (TNF) and interleukin-1 (IL-1). In addition, AGE modified proteins induce monocytes/macrophages to generate growth factors-platelet derived growth factor $(\mathrm{PDGF})^{6}$ and insulin-like growth factor I (IGF-I) ${ }^{7}$ - by the AGE receptor system. These cytokines and growth factors secreted by lung macrophages are thought to play cardinal roles in the pathogenesis of pulmonary fibrosis.

In this study, we examined the immunohistochemical localisation of AGE modified proteins in the remodelling lesions of pulmonary fibrosis, using a monoclonal antibody specific for AGE (6D12). ${ }^{8}$ We showed accumulation of AGE modified proteins in lung macrophages in diffuse alveolar damage and remodelling lesions of pulmonary fibrosis, suggesting that chemical modification by AGE could be a biomarker of oxidative stress or might play some role in pulmonary fibrosis.

\section{Methods}

MONOCLONAL ANTI-AGE ANTIBODY

A monoclonal mouse anti-AGE antibody (6D12) was produced by immunisation of $\mathrm{BALB} / \mathrm{c}$ mice with AGE bovine serum albumin (AGE-BSA) as described elsewhere. ${ }^{4}$ Briefly, splenic lymphocytes from Balb/c mouse immunised with AGE-BSA were fused to myeloma P3U1 cells. The hybrid cells were screened to select cell lines positive for AGE-BSA but negative for BSA. One cell line, termed 6D12 cell, was selected and further purification by protein A affinity chromatography to IgG1 was 


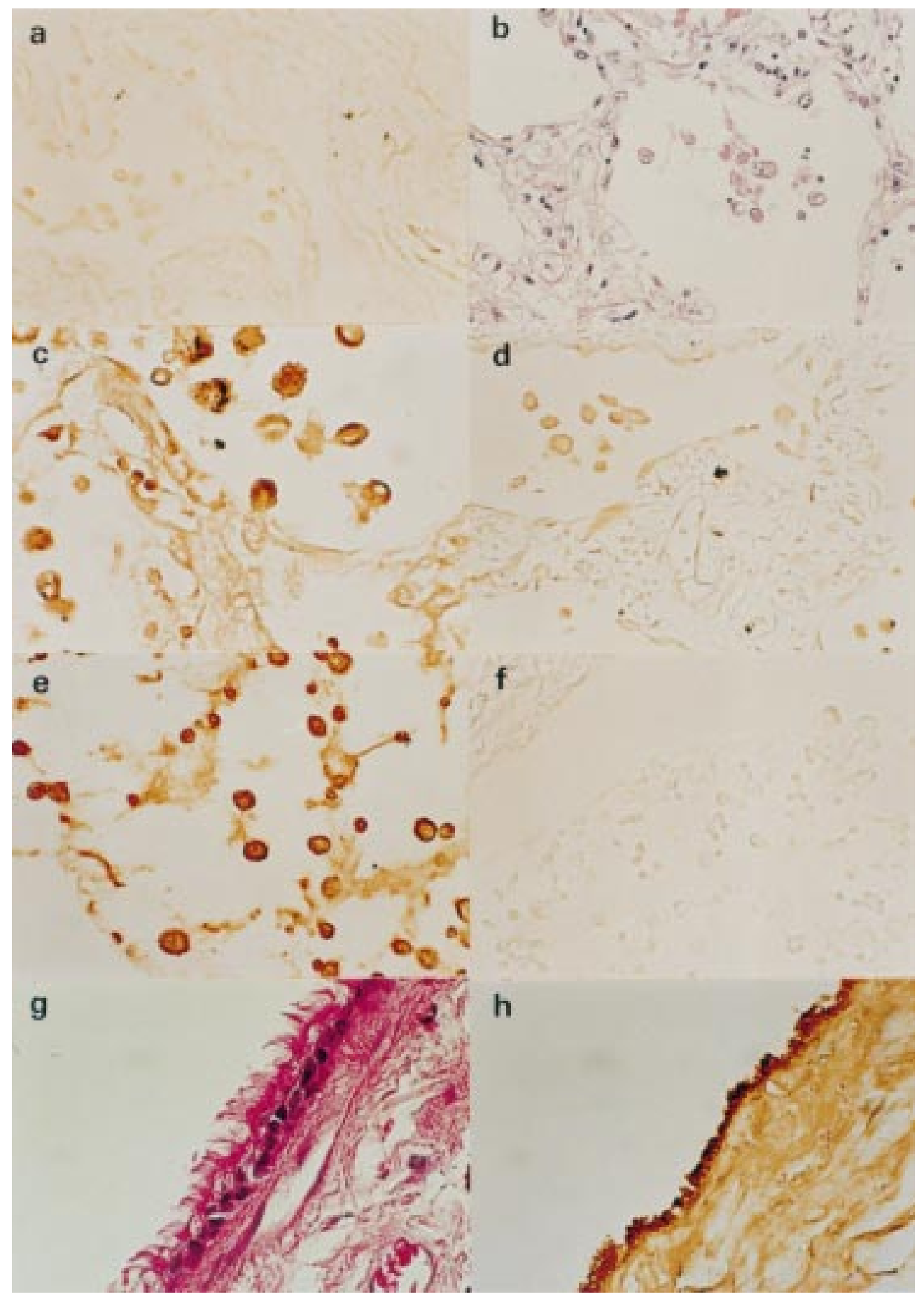

Figure 1 Immunohistochemistry of lung specimens obtained from cases with normal parenchyma, diffuse alveolar damage, and idiopathic pulmonary fibrosis. (a) 6D12 immunoperoxidase staining of normal lung parenchyma showing no immunoreactivity on macrophages and alveolar epithelium $(\times 233)$. (b) Haematoxylin and eosin $(H$ and $E$ ) staining of a lung specimen obtained from a case with diffuse alveolar damage showing interstitial oedema and alveolar macrophages ( $\times 233)$. (c) 6D12 immunoperoxidase staining of the same specimen as in (b). Note that the 6D12 positive cells are macrophages $(\times 467)$. (d) Negative control for immunoperoxidase staining of the same lung specimen as in $(c)$, in which primary antibody was replaced with non-specific mouse IgG1 (×233). (e) 6 D12 immunoperoxidase staining of a lung specimen obtained from a case with idiopathic pulmonary fibrosis $(\times 467)$. Note that the 6 D12 positive cells are alveolar macrophages. $(f)$ Negative control for immunoperoxidase staining of the same specimen as in (e), in which primary antibody was replaced with non-specific mouse IgG1 (×233). (g) $H$ and $E$ staining of the same specimen as in (e), showing columnar cells (metaplastic epithelium) in the honeycomb lesion $(\times 467)$. (h) 6 D12 immunoperoxidase staining of the same specimen as in $(\mathrm{g})$, showing $6 \mathrm{D} 12$ positive metaplastic epithelium $(\times 467)$.

performed. 6D12 reacts not only with AGE proteins such as AGE-BSA, AGE human serum albumin, AGE human haemoglobin, and AGE collagen, but also with AGE preparation obtained either from lysine derivatives or monoaminocarboxylic acids, whereas it does not react with unmodified counterparts. ${ }^{4}$ Thus $6 \mathrm{D} 12$ reacts with the main AGE structure common to all AGE preparations. ${ }^{8}$ A recent study showed that the epitope of $6 \mathrm{D} 12$ is an $\mathrm{N}$-(carboxymethyl)lysine-protein (CML) adduct with a carbonyl group important for 
immunological recognition of AGE modified proteins. $^{9}$

CASES

Seventeen lung samples obtained from necropsies were analysed. Seven were of idiopathic pulmonary fibrosis and three of diffuse alveolar damage. These diagnoses were made on the basis of clinical course, chest $x$ ray examinations, pulmonary function tests, and postmortem histopathology. Seven cases with normal parenchyma comprised the control group. Causes of death in the control group were as follows: two cases of renal cell carcinoma and one case each of acute myocardial infarction, malignant lymphoma, pancreatitis, purulent inflammation, and liver cirrhosis. Three cases from the control group had diabetic mellitus. The range of time from death to obtaining the specimens in each group was as follows: idiopathic pulmonary fibrosis, 2-5 hours; diffuse alveolar damage, $0.5-22.3$ hours; normal control, 1.2-4.5 hours.

\section{IMMUNOHISTOCHEMISTRY}

Each specimen was fixed with buffered formalin and embedded in paraffin wax. Immunohistochemical detection for AGE was done by the immunoperoxidase technique. Briefly, the tissue sections were dewaxed and treated with 3\% hydrogen peroxide in methanol to inhibit endogenous peroxidase. The sections were then preblocked and incubated with $1.2 \mu \mathrm{g} / \mathrm{ml}$ of a monoclonal mouse antibody for AGE (6D12) overnight, followed by a biotinylated antimouse IgG antibody (Nichirei Inc, Tokyo, Japan). After incubation with streptavidin conjugated with horseradish peroxidase, specifically bound antibodies were visualised using peroxidase substrate, 3,3'-diamino benzidine (DAB; Vector Laboratories Inc, Burlingame, California, USA). To compare the distribution and intensity of immunostaining, all samples were studied in an identical fashion using an identical concentration of antibody, identical batches of reagents, and identical development times. Immunohistochemical controls consisted of substitution of purified mouse IgG1 (Sigma, Missouri, USA) for the primary antibody.

EVALUATION OF IMMUNOSTAINING

The degree of immunostaining was evaluated by an arbitrary semiquantitative scale, as described previously ${ }^{10}$ : for alveolar macrophages, $-=$ negative or only a small subpopulation (less than $10 \%$ of cells) faintly stained; + $=$ positive, more than half of cells were stained; $++=$ strongly positive, most cells (more than two thirds of cells) were stained. For bronchiolar epithelium, metaplastic epithelium, and smooth muscle cells, $-=$ no staining or the same level of background staining; $\pm=$ faintly positive or only a small subpopulation was stained; $+=$ positive, most cells were stained; $++=$ strong positive means marked staining homogeneously.

Non-parametric comparison of the degree of immunoreactivity of alveolar macrophages between the control and idiopathic pulmonary fibrosis groups was made by the MannWhitney U test. Probability (p) values less than 0.05 were taken as significant.

\section{Results}

Table 1 summarises the findings of the immunohistochemical studies. The lung specimens with normal parenchyma obtained from all but two cases without pulmonary diseases were negative for $6 \mathrm{D} 12$ immunohistochemistry. Only two cases with normal parenchyma showed positive immunoreactivity on macrophages (one case had diabetes mellitus and the other was not diabetic). Two of three cases with diabetes mellitus were negative for $6 \mathrm{D} 12$. No lung specimens with normal parenchyma expressed immunoreactivity on the bronchiolar epithelium.

In marked contrast, strong 6D12 immunoreactivity was observed on alveolar macrophages from all seven cases with idiopathic pulmonary fibrosis (table 1). The earliest lesion of pulmonary fibrosis detected histopathologically is a macrophage-rich fibrinous exudate within alveoli associated with epithelial injury, which is called diffuse alveolar damage. Strong 6D12 immunoreactivity was observed on alveolar macrophages from all three cases of diffuse alveolar damage. The bronchiolar epithelium from some cases with diffuse alveolar damage and the metaplastic columnar epithelium of pulmonary fibrosis also showed positive $6 \mathrm{D} 12$ staining on immunohistochemistry. Hyperplastic smooth muscle cells and interstitium were positive in some cases of pulmonary fibrosis.

Typical immunohistochemical findings from cases with diffuse alveolar damage and idiopathic pulmonary fibrosis are shown in fig 1 . Positive immunoreactivity was observed on alveolar macrophages and on the metaplastic epithelium in the honeycomb lesions.

\section{Discussion}

Our study showed the presence of advanced glycation end products in all specimens from cases with pulmonary fibrosis and diffuse alveolar damage. However, the lung specimens from normal parenchyma showed positive AGE immunoreactivity on macrophages from only two of seven cases. Strong 6D12 immunoreactivity was observed on alveolar macrophages from all cases with pulmonary fibrosis. In marked contrast, most alveolar macrophages from control cases were negative for $6 \mathrm{D} 12$, which suggests that $6 \mathrm{D} 12$ immunoreactivity is not a marker for alveolar macrophages. Our findings also raise the interesting possibility that AGE modified proteins could be a biomarker of oxidant stress or might be involved in the pathogenesis of pulmonary fibrosis.

In the Maillard reaction, proteins react with glucose to form stable Amadori products through Schiff base adducts. Upon long term incubation, these early products are converted to AGE, which are characterised by a brown colour, autofluorescent properties, and interor intramolecular cross linking. ${ }^{12}$ Immunohistochemical studies using anti-AGE antibodies including $6 \mathrm{D} 12$ have shown the presence of 
AGE modified proteins in several tissues including atherosclerotic lesions of arterial walls, ${ }^{3411}$ human lens proteins, ${ }^{12}$ and amyloid fibril deposits in patients with haemodialysis related amyloidosis. ${ }^{13}{ }^{14}$ The presence of AGE has also been demonstrated in lens, aorta, and kidney of streptozocin induced diabetic rats. ${ }^{15-17}$ These findings from immunological studies with anti-AGE antibodies have suggested a potential involvement of AGE modification in disease processes such as diabetic complications and atherosclerosis.

Pulmonary fibrosis is characterised by the presence of chronic inflammation and increased deposition of collagen in lung parenchyma. The key cellular participants in the pathogenesis of pulmonary fibrosis have not be defined. However, lung macrophages are assumed to be one of the major participants in these fibroproliferative processes, because they secrete various inflammatory cytokines such as TNF and growth factors including platelet derived growth factor (PDGF), IGF-I, and granulocyte monocyte colony stimulating factor (GM-CSF). ${ }^{18-21}$

Macrophages play an essential role in eliminating AGE modified proteins. AGE modified proteins are biologically recognised by AGE binding proteins or AGE receptors of the cell surface membrane. ${ }^{22}{ }^{23}$ Recent experiments with Chinese hamster ovary cells overexpressing the macrophage scavenger receptor (MSR) or with peritoneal macrophages isolated from MSR-knockout mice have indicated that MSR plays a major role as the AGE receptor in the endocytic uptake of AGE modified proteins by macrophages. ${ }^{2425}$ The other AGE receptorthe receptor for AGE (RAGE)-has been reported to mediate macrophage activation and induce the production and secretion by macrophages of several cytokines such as TNF and IL- $1^{5}$ and growth factors such as PDGF ${ }^{6}$ and IGF-1. ${ }^{7}$ Moreover, it is suggested that the macrophage AGE receptor stimulates the cells to release GM-CSF, which induces macrophage growth in an autocrine or paracrine fashion. ${ }^{26}$ These AGE induced cellular responses in macrophages raise the possibility that AGE modified proteins taken up by the AGE receptors of macrophages may play a role in the pathogenesis of pulmonary fibrosis.

Further, reactive oxygen intermediates are known to be implicated in the pathogenesis of pulmonary injury, diffuse alveolar damage, and pulmonary fibrosis. AGE modified proteins have been shown to generate reactive oxygen intermediates. ${ }^{27}{ }^{28}$ Reactive oxygen intermediates are also involved in the formation of AGE structures such as carboxymethyllysine (CML). ${ }^{29} \mathrm{CML}$ is thus also considered to be a potential biomarker of oxidative damage of tissue proteins in vivo. ${ }^{29}{ }^{30}$ The immunological demonstration of CML modified proteins in several animal and human tissues ${ }^{4-14}{ }^{17}$ indicates that reactive oxygen intermediates are generated in situ. Taken together, these results suggest that the interaction of AGE modified proteins with macrophages might induce oxidant stress, which could also contribute to the development of pulmonary fibrosis.
In addition, we found positive AGE staining on bronchiolar and metaplastic epithelium in cases of diffuse alveolar damage and idiopathic pulmonary fibrosis. No previous study has looked for AGE receptors on bronchial epithelium. Although we are not able to determine the significance of AGE in bronchiolar and metaplastic epithelium in this study, the presence of AGE modified protein in these epithelial cells in cases of diffuse alveolar damage and idiopathic pulmonary fibrosis suggests a potential role as a biomarker of oxidative damage to tissue proteins.

We found positive AGE staining on alveolar macrophages in normal parenchyma obtained from two cases, one of which had diabetes mellitus. Although positive AGE staining might be related to the presence of diabetes, macrophages in the other two cases of diabetes were found to be negative for AGE.

In conclusion, this study is the first to show the presence of AGE modified proteins on alveolar macrophages and bronchiolar epithelium in cases of diffuse alveolar damage and pulmonary fibrosis. The exact role of the AGE modified proteins that accumulate in the lung remain to be elucidated.

Supported by research funds from the Naito Research Foundation, in part by grants from the Ministry of Education, Science and Culture of Japan (C2-09670603) and the Smoking Research Foundation

1 Maillard LC. Action des acides amines sur les sucres; formation des mélanoidines par voie méthodique. C R Acad Sci Paris 1912;154:66-8

2 Brownlee M, Cerami A, Vlassara H. Advanced glycosylation end products in tissue and the biochemical basis of diabetic complications. N Engl f Med 1988;318:1315-21.

3 Nakamura Y, Horii Y, Nishino T, et al. Immunohistochemical localization of advanced glycosylation endproducts in coronary atheroma and cardiac tissue in diabetes mellitus. Am 7 Pathol 1993;143:1649-56.

4 Kume S, Takeya M, Mori T, et al. Immunohistochemical and ultrastructural detection of advanced glycation end products in atherosclerotic lesions of human aorta with a novel specific monoclonal antibody. Am F Pathol 1995;147: 654-67.

5 Vlassara $\mathrm{H}$, Brownlee M, Manogue KR, et al. Cachectin/ TNF and IL-1 induced by glucose-modified proteins: role in normal tissue remodeling. Science 1988;240:1546-8.

6 Kirstein M, Brett J, Radoff S, et al. Advanced protein glycosylation induces transendothelial human monocyte chemotaxis and secretion of platelet-derived growth factor: role in vascular disease of diabetes and aging. Proc Natl Acad Sci USA 1990;87:9010-4

7 Kirstein M, Aston C, Hintz R, et al. Receptor-specific induction of insulin-like growth factor $\mathrm{I}$ in human monocytes by advanced glycosylation end productmodified proteins. F Clin Invest 1992;90:439-46.

8 Horiuchi S, Araki N, Morino Y. Immunohistochemical approach to characterized advanced glycation end products of the Maillard reaction: evidence for the presence of a common structure. F Biol Chem 1991;266:7329-32.

9 Ikeda K, Higashi T, Sano H, et al. N-(carboxymethyl)lysine protein adduct is a major immunological epitope in proteins modified with advanced glycation end products of proteins modified with advanced glycation end products

10 Matsuse T, Ikegami A, Ohga E, et al. Expression of immunoreactive activin A protein in remodeling lesions associated with interstitial pulmonary fibrosis. Am f Pathol 1996;148:707-13

11 Horiuchi S. AGE-modified proteins and their potential relevance to atherosclerosis. Trends Cardiovasc Med 1996;6: 163-8.

12 Araki N, Ueno N, Chakrabarti B, et al. Immunohistochemical evidence for the presence of advanced glycation end products in human lens protein and its positive correlation with aging. F Biol Chem 1992;267:10211-14

13 Miyata $T$, Oda $\mathrm{O}$, Inagi $\mathrm{R}$, et al. $\beta 2$-Microglobulin modified with advanced glycation end products is a major component of hemodialysis-associated amyloidosis. $\mathcal{7}$ Clin Invest 1993;92:1243-52.

14 Miyata $T$, Taneda S, Kawai R, et al. Identification of pentosidine as a native structure for advanced glycation end products in $\beta 2$-microglobulin-containing amyloid fibrils in products in $\beta 2$-microglobulin-containing amyloid fibrils in Sci USA 1996;93:2353-8.

15 Nakayama H, Mitsuhashi T, Kuwajima S, et al. Immunohistochemical detection of advanced glycation end products in 
lens crystalline from streptozocin-induced diabetic rats. Diabetes 1993;42:345-50.

16 Mitsuhashi $\mathrm{T}$, Nakayama $\mathrm{H}$, Itoh $\mathrm{T}$, et al. Immunohistochemical detection of advanced glycation end products in renal cortex from STZ-induced diabetic rats. Diabetes 1993;42:826-32.

17 Meng J, Sakata N, Takebayashi S, et al. Advanced glycation end-products of the Maillard reaction in aortic pepsininsoluble collagen from diabetic rats. Diabetes 1996;45: 1037-43

18 Martinet Y, Rom WN, Grotendorst GR, et al. Exaggerated spontaneous release of platelet-derived growth factor by alveolar macrophages from patients with idiopathic pulmonary fibrosis. N Engl f Med 1987;317:202-9.

19 Rom WN, Bassett P, Fell GA, et al. Alveolar macrophages release an insulin-like growth factor I-type molecule. $\mathcal{F}$ Clin Invest 1988;82:1658-93.

20 Kelley J. Cytokines of the lung. Am Rev Respir Dis 1990;141: $765-88$.

21 Gauldie J, Jordana M, Cox G. Cytokines and pulmonary fibrosis. Thorax 1993;48:931-5.

22 Vlassara H, Brownlee M, Cerami A. Novel macrophage receptor for glucose-modified proteins is distinct from previously described scavenger receptors. F Exp Med 1986;164 1301-9.

23 Neeper M, Schmidt AM, Brett J, et al. Cloning and expression of a cell surface receptor for advanced glycosylation end products of proteins. F Biol Chem 1992;267:149985004

24 Araki N, Higashi T, Mori T, et al. Macrophage scavenger receptor mediates the endocytic uptake and degradation of advanced glycation end products of the Maillard reaction. Eur F Biochem 1995;230:408-15.

25 Suzuki H, Kurihara Y, Takeya $M$, et al. A role for macrophage scavenger receptors in atherosclerosis and susceptibility to infection. Nature 1997;386:292-6.

26 Yui S, Sasaki T, Araki N, et al. Induction of macrophage growth by advanced glycation end products of the Maillard reaction. F Imunol 1994;152:1943-9.

27 Yan SD, Schmidt AM, Anderson GM, et al. Enhanced cellular oxidant stress by the interaction of advanced glycation end products with their receptors/binding proteins. $\mathcal{F}$ Biol Chem 1994;269:9889-97.

28 Schmidt AM, Hori O, Brett J, et al. Cellular receptors for advanced glycation end products. Implications for induction of oxidant stress and cellular dysfunction in the pathogenesis of vascular lesions. Arterioscler Thromb 194;14: 1521-8.

29 Nagai R, Ikeda $\mathrm{K}$, Higashi $\mathrm{T}$, et al. Hydroxyl radical mediates $\mathrm{N}^{\varepsilon}$-carboxymethyl)lysine formation from amadori product. Biochem Biophys Res Commun 1997;234:16772 .

30 Baynes JW. Role of oxidative stress in development of complications in diabetes. Diabetes 1991;40:405-12. 